
И ПОДАГРУ

\author{
(с) Т.С. Паневин ${ }^{1,2,3 *}$, М.С. Елисеев ${ }^{1}$, М.В. Шестакова²
}

\begin{abstract}
'Научно-исследовательский институт ревматологии им. В.А. Насоновой, Москва, Россия
${ }^{2}$ Национальный медицинский исследовательский центр эндокринологии, Москва, Россия

${ }^{3}$ Консультативно-диагностический центр Генерального штаба Вооруженных сил Российской Федерации, Москва,

Россия
\end{abstract}

Подагра является системным аутовоспалительным тофусным заболеванием и характеризуется отложением кристаллов моноурата натрия (МУН) с последующим развитием воспаления у лиц с гиперурикемией (ГУ). Помимо генетических, факторами риска развития ГУ и подагры являются многие модифицируемые факторы, среди которых метаболический синдром и ожирение, а их коррекция - важнейшая составляющая успешного контроля над заболеванием. Ожирение может изменять клиническую картину подагры, характеризующуюся более ранним началом и бо́льшим числом пораженных суставов. Потеря веса способствует снижению уровня мочевой кислоты (МК) сыворотки. Бариатрическая/метаболическая хирургия значительно сокращает частоту развития коморбидных ожирению состояний и смертность и на сегодняшний день является наиболее эффективным методом борьбы с ожирением. Помимо улучшения состояния здоровья, важная цель бариатрической хирургии - повысить качество жизни. Бариатрические операции могут предотвращать развитие ГУ у субъектов с исходно нормальным уровнем сывороточной МК до оперативного вмешательства. Динамика концентрации МК в сыворотке крови может быть предиктором успешно проведенного бариатрического лечения и помочь в прогнозировании выраженности послеоперационной потери веса. Снижение массы тела после проведения бариатрических операций приводит к уменьшению МК в отдаленном периоде, однако наблюдается высокая частота острых приступов подагры в раннем послеоперационном периоде. Таким образом, бариатрическая хирургия оказывает значимое влияние на ГУ и подагру и может быть эффективным методом лечения данного заболевания.

КЛЮЧЕВЫЕ СЛОВА: ожирение; подагра; гиперурикемия; мочевая кислота; сахарный диабет 2 типа; бариатрическая хирургия.

\title{
THE EFFECT OF BARIATRIC SURGERY ON PURINE METABOLISM AND GOUT
}

(c) Taras S. Panevin ${ }^{1,2,3 *}$, Maxim S. Eliseev ${ }^{1}$, Marina V. Shestakova ${ }^{2}$

'V.A. Nasonova Research Institute of Rheumatology, Moscow, Russia

2Endocrinology Research Centre, Moscow, Russia

${ }^{3}$ Advisory and Diagnostic center of the General Staff of the Armed Forces of the Russian Federation, Moscow, Russia

Gout is an auto-inflammatory tophaceous disease characterized by the deposition of crystals of monosodium urate and developing in connection with this inflammation in people with hyperuricemia (HU). Except to genetic factors, risk factors for the development of HU and gout are many modifiable factors, including metabolic syndrome and obesity, and their correction is the most important component of successful disease control. Obesity can change the clinical picture of gout, characterized by an earlier onset, a large number of affected joints. Weight loss helps reduce uric acid (UA) serum levels. Bariatric / metabolic surgery significantly reduces the incidence of comorbid obesity and mortality, and is by far the most effective way to combat obesity. In addition to improving health, an important goal of bariatric surgery is to improve the quality of life. Bariatric surgery can prevent the development of HU in subjects with an initially normal level of serum UA before surgery. The dynamics of the concentration of UA in serum can be a predictor of successful bariatric surgery and help in predicting the severity of postoperative weight loss. The decrease in body weight after bariatric surgery leads to a decrease in UA in the long-term postoperative period, however, there is a high frequency of acute gout attacks in the early postoperative period. Thus, bariatric surgery has a significant effect on $\mathrm{HU}$ and gout and can be an effective treatment for this disease.

KEYWORDS: obesity; gout; hyperuricemia; uric acid; type 2 diabetes; bariatric surgery.

*Автор, ответственный за переписку / Corresponding author. 


\section{ВВЕДЕНИЕ}

Подагра - системное аутовоспалительное тофусное заболевание, характеризующееся отложением кристаллов моноурата натрия (МУН) и развивающимся в связи с этим воспалением у лиц с гиперурикемией (ГУ), обусловленной внешнесредовыми и/или генетическими факторами [1, 2]. Подагра является одним из самых древних и известных ныне заболеваний, реальное описание которого насчитывает несколько тысячелетий. В основе развития подагры лежит накопление кристаллов МУН [3, 4], которое происходит, когда уровень сывороточной мочевой кислоты превышает «порог насыщения», составляющий более 404 мкмоль/л (>6,8мг/дл) при физиологической температуре [5].

Формирование кристаллов in vivo возможно и при меньшем сывороточном уровне МК (>360 мкмоль/л (>6,0 мг/дл)), поэтому именно данный показатель следует считать соответствующим ГУ [6]. В большинстве случаев ГУ протекает асимптоматически, однако примерно в 20\% случаев она приводит к подагре, являясь единственным доказанным на сегодняшний день патогенетическим фактором риска ее возникновения [7, 8]. Зависимость между уровнем МК сыворотки и риском развития подагры прямая, но не линейная, и даже при максимально высоких значениях уровня МК (>10 мг/дл) 15-летний риск не превышает 50\% [9]. Тем не менее даже в случае бессимптомного течения (при отсутствии клинических проявлений отложения кристаллов МУН) ГУ часто ассоциируется с наличием кристаллов МУН [4]. Кроме того, как подагра, так и ГУ ассоциированы с развитием артериальной гипертензии (АГ) и других сердечно-сосудистых заболеваний, развитием цереброваскулярных заболеваний и снижением почечной функции, ожирением и метаболическим синдромом. Повышение уровня МК на каждый 1 мг/дл приводит к возрастанию риска общей смерти на 9\% и риска развития СС3 на 20\% [10-12].

С течением времени, при накоплении кристаллов МУН возможно развитие острого подагрического воспаления, которое может протекать в виде артрита, а также бурсита и тендинита. Согласно современным представлениям, выделяют доклиническую и клиническую стадию подагры. Для доклинической стадии характерна бессимптомная ГУ, которая сменяется ГУ с отложением кристаллов МУН. Возможно также отложение кристаллов МУН в отсутствие ГУ. Клиническая стадия включает острый и хронический подагрический артрит, тофусную подагру, а также подагру с наличием костных эрозий [13].

Диагноз подагры устанавливается на основании классификационных критериев Американской коллегии ревматологов (ACR, EULAR) 2015 г., в которых учитывается клиническая картина (характеристика приступов артрита, наличие тофусов), уровень МК крови, наличие симптомов подагры по данным инструментальных исследований (УЗИ, рентгенография, двухэнергетическая компьютерная томография (ДЭКТ)). Золотым стандартом подтверждения клинического диагноза является обнаружение кристаллов МУН в синовиальной жидкости или тофусных массах при помощи поляризационной микроскопии [14].

Распространенность подагры в развитых странах в последние десятилетия увеличивается, достигая, например, в США, 4\% среди взрослого населения, что обусловлено ростом распространенности ожирения и изменением рациона питания $[15,16]$. Учитывая эпидемическую тенденцию избыточного веса и ожирения в развитых странах, распространенность подагры в целом, вероятно, также продолжит расти [17]. Эпидемиология подагры варьирует в зависимости от возраста, расы и пола: наиболее часто заболевают мужчины старше 40 лет, в пожилом и старческом возрасте половые различия в распространенности заболевания практически нивелируются [18]. Большое значение в генезе ГУ и подагры имеет генетическая предрасположенность, обусловленная прежде всего недостаточной экскрецией почками MK, преимущественно за счет увеличения ее реабсорбции. Обнаружено несколько белков-транспортеров, ответственных за реабсорбцию и экскрецию МК в почках: URAT1, OAT4, OAT10, GLUT9 и ABCG2 [19].

B меньшей степени MK накапливается вследствие снижения ее экскреции через кишечник. В желудочно-кишечном тракте МК поступает из кровотока, желчи, слюны и пептических секретов и обычно полностью реабсорбируется транспортерами кишечника. Мутации гена уратного транспортера ABCG2, который представлен как в почках, так и в желудочно-кишечном тракте, связаны с повышением МК в сыворотке крови $[20,21]$. Помимо генетических, факторами риска развития ГУ и подагры являются многие модифицируемые факторы, среди которых основные - АГ, метаболический синдром (MC), хроническая болезнь почек, прием диуретиков и аспирина, ожирение, злоупотребление алкоголем, менопауза, и именно их коррекция - важнейшая составляющая успешного контроля над заболеванием [22-24].

Одним из наиболее эффективных методов достижения устойчивого снижения массы тела является бариатрическая хирургия, однако ее влияние на пуриновый обмен изучено в меньшей степени. При подготовке данного обзора поиск статей осуществлялся в базе данных Medline (PubMed) по ключевым словам: «Gout», «Hyperuricemia», "Uric acid»», «Bariatric surgery», «Obesity», «Type 2 diabetes». Для повышения специфичности и ограничения чувствительности поиска использовался оператор «AND». Поиск проводился также по спискам литературы анализируемых статей. Из 186 найденных статей были выбраны 18 работ, соответствующих задачам нашего обзора.

\section{ВЛИЯНИЕ ОЖИРЕНИЯ НА РАЗВИТИЕ И КЛИНИЧЕСКИЕ ПРОЯВЛЕНИЯ ПОДАГРЫ}

У пациентов с ожирением метаболизм пуринов может быть нарушен пищевыми привычками, основанными на переедании, преобладании в рационе жирной и богатой пуринами пищи, употреблении алкоголя и фруктозосодержащих напитков. Так, метаболизм фруктозы осуществляется посредством ферментативного каскада, начинающегося с форфорилирования, при котором потребность в фосфатах восполняется путем деградации аденозинтрифосфата до аденозиндифосфата и аденозинмонофосфатная и далее - до инозина, гипоксантина и, наконец, мочевой кислоты [25]. Алкоголь угнетает почечную экскрецию урата путем увеличения выработки лактата, а также за счет собственного высокого содержания 
пуринов в некоторых алкогольных напитках, особенно в пиве [26]. Несмотря на тот факт, что избыточное потребление пуринов значительно повышает риск развития ГУ, взаимосвязь между ожирением и ГУ не ограничивается пищевыми привычками, т.к. избыточный вес часто ассоциирован с инсулинорезистентностью (ИР) и повышением продукции лептина, снижающего выведение МК. Сопряженная с ИР гиперинсулинемия уменьшает почечную экскрецию МК, приводя к ГУ [27]. Гипертриглицеридемия, являющаяся компонентом МС, может способствовать усилению синтеза пуринов de novo, увеличивая продукцию МК [28, 29].

Напротив, потеря веса приводит к снижению уровня МК сыворотки, главным образом - за счет увеличения выведения ее почками и частично - за счет снижения выработки урата. Показано, что фракционная экскреция МК была значительно ниже среди людей с ожирением по сравнению с контрольной группой с нормальным индексом массы тела (ИМТ) (4-5\% против 11-12\% соответственно) [30], а снижение веса с помощью диеты и физических упражнений приводило к нормализации фракционного выведения почками мочевой кислоты.

S. Neto и коллеги изучили распространенность ГУ у 420 пациентов с морбидным ожирением. Общая распространенность ГУ составила 34,28\%. Мужчины с ГУ чаще страдали сахарным диабетом 2 типа (СД2) и имели более высокий уровень глюкозы в плазме натощак [31]. Третье национальное исследование состояния здоровья и питания (NHANES) подтверждает, что распространенность различных компонентов MC - гипертонии, абдоминального ожирения, дислипидемии (особенно гипертриглицеридемии) и СД2 выше при подагре, чем у пациентов без нее [32]. Распространенность МС достаточно высока и составляет 14-24\%. Лидируют по распространенности МС США, относительно часто синдром встречается в северных европейских странах. По результатам исследования, проведенного в Финляндии и Швеции, МС при отсутствии нарушений углеводного обмена имеют 10\% женщин и 15\% мужчин, при повышенной гликемии натощак и/или нарушении толерантности к глюкозе (НТГ) - 42 и 64\% соответственно, а при СД2 - 78 и 84\% [33]. По данным отечественных исследований, распространенность МС в Российской Федерации составляет около 40\% [34].

Висцеральное ожирение представляет собой специфический маркер риска подагры у пациентов с МС [35]. Жировая ткань фактически влияет на различные метаболические пути через эндокринную регуляцию, что способствует развитию СД2, сердечно-сосудистых, а также аутоиммунных заболеваний и поражений костно-мышечной системы. Вероятно, морбидное ожирение увеличивает внутрисуставные концентрации протеолитических ферментов, таких как матриксные металлопротеиназы (MMP), и нарушает метаболизм костной и хрящевой тканей, увеличивая склонность к остеоартриту [36].

Кроме того, жировая ткань реализует свою эндокринную функцию, в том числе путем продукции таких веществ, как лептин, резистин, интерлейкин-6 (ИЛ-6), фактор некроза опухоли а (ФНОа), адипонектин. Постоянное высвобождение ИЛ-6 и ФНОа из жировой ткани и последующее провоспалительное состояние связаны с гиперинсулинемией и инсулинорезистентностью (ИР), которые, вероятно, влияют на почечный метаболизм МК за счет увеличения ее реабсорбции [37]. ИР также может способствовать повышенной реабсорбции натрия в почках, активации симпатической нервной системы и ремоделированию сосудов, провоцируя развитие АГ, которая, в свою очередь, может способствовать развитию ГУ через нарушение почечной гемодинамики [38].

Ожирение может изменять клиническую картину подагры, что проявляется более ранним началом, бо́льшим числом пораженных суставов [39-41]. Необходимость коррекции ожирения и модификации образа жизни постулируется всеми наиболее значимыми рекомендациями по лечению подагры, но, тем не менее, возможность достижения целевого уровня МК только путем соблюдения низкопуриновой диеты невелика.

P. Dessein и коллеги исследовали влияние низкопуриновой диеты на улучшение чувствительности к инсулину у 13 человек с плохо контролируемой подагрой и средним ИМТ 30,5 кг/м². Участники достигли средней потери веса на 7,7 кг, кроме того, наблюдалось достоверное снижение частоты острых приступов артрита, а также уровня МК сыворотки с 0,57 ммоль/л до 0,47 ммоль/л. Тем не менее ни одному из участников с исходным уровнем МК сыворотки выше целевого (>0,36 ммоль/л) не удалось достичь нормального значения [28]. По данным крупного 7-летного ретроспективного исследования, ни один из 18 пациентов с подагрой, длительно соблюдавших низкопуриновую диету без сопутствующего приема уратснижающих препаратов, не достиг целевого уровня МК сыворотки, хотя это и снижало риск приступов артрита, образования тофусов, сопутствующих заболеваний [42]. По данным H. Ellmann и соавт., коррекция образа жизни, включавшая соблюдение диеты 24 пациентами с нетяжелой подагрой на протяжении 18 месяцев, приводила к незначительному снижению сыво-

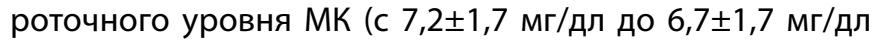
(т.е. на 0,5 22,0 мг/дл, или 30 ммоль/л)), но в 58\% даже этого было достаточно для полного растворения имеющихся отложений МУН в суставах стоп по результатам двухэнергетической компьютерной томографии (ДЭКТ) [43]. Однако результаты анализа ключевых работ, посвященных влиянию снижения массы тела на сывороточный уровень МК, показали, что только в 6 из 10 рассматриваемых исследований снижение уровня было достоверным, но и в этом случае данные изменения можно отчасти объяснить плейотропными эффектами принимаемых пациентами препаратов (например, метформина), используемых в ряде работ [44]. Кроме того, назначение диеты сопряжено с множеством сложностей, предопределяющих невозможность и нежелание ее длительного соблюдения и потому редко реализуется [45]. Медикаментозные методы лечения ожирения зачастую не способны помочь достичь стойкого желаемого эффекта в отношении как собственно массы тела, так и метаболических нарушений, ему сопутствующих [46]. В таких случаях, при невозможности адекватной коррекции массы тела при назначении диеты и приеме лекарственных препаратов, одним из методов лечения у пациентов с морбидным ожирением рассматривается бариатрическая хирургия, приводящая к более стабильной потере веса, улучшению коморбидных состояний и повышению качества жизни [47]. 


\section{БАРИАТРИЧЕСКАЯ ХИРУРГИЯ И ПОДАГРА}

Бариатрическая (метаболическая) хирургия значительно сокращает частоту развития коморбидных ожирению состояний и смертность и на сегодняшний день является наиболее эффективным методом борьбы с ожирением $[48,49]$. Необходимость в ней возникает при отсутствии достижения целевых показателей массы тела путем модификации образа жизни, а также при неэффективности медикаментозного лечения ожирения или наличия к нему противопоказаний [50]. Основные показа-

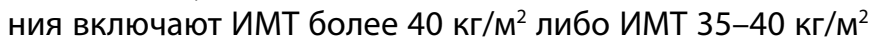
при наличии коморбидных состояний, коррекция которых возможна при снижении массы тела (СД2, заболевания сердечно-сосудистой системы, поражение суставов, синдром обструктивного апноэ сна) [51].

Первым рандомизированным контролируемым исследованием стала работа Р. O'Brien с соавт. [52], где 80 пациентам была назначена либо комбинация низкокалорийной диеты, лекарственной терапии (орлистат) и изменения образа жизни в течение 24 месяцев (нехирургическая группа), либо лапароскопическое установление регулируемого желудочного бандажа (LAP-BAND System, INAMED Health, Santa Barbara, California) (хирургическая группа). Помимо снижения массы тела к концу исследования на 21,6\% от исходной, что было почти в 4 раза больше, чем в группе сравнения (5,5\%), хирургическое лечение отождествлялось с лучшей коррекцией нарушений липидного обмена, обмена глюкозы, снижением показателей АД. Динамика показателей, отражающих качество жизни, измеренная при помощи опросника SF-36, в случае хирургического лечения для параметров как физического, так и ментального здоровья также была лучшей. И хотя сывороточный уровень МК в данном исследовании не оценивался, изменение ряда параметров могло иметь ключевое значение для коррекции урикемии и профилактики развития подагры.

Уменьшение уровня МК наблюдается при стабильной потере веса, снижении АД, улучшении почечной функции [29], снижении ИР [53], нормализации уровня триглицеридов в сыворотке крови [54] и, возможно, качественных изменениях рациона питания. Помимо этого, большое значение может иметь угнетение выраженности хронического воспаления, достигаемого после потери веса [55]. Так, N. Dalbeth и соавт. показали достоверное уменьшение уровней ИЛ-1 $\beta$, ИЛ-8 и ИЛ-6 в крови после бариатрических операций, имеющих ключевое значение для развития микрокристаллического воспаления [56]. Таким образом, бариатрическое оперативное вмешательство, вероятно, приводит к ослаблению реактивности на кристаллы МУН, что способствует снижению частоты приступов подагры. По данным крупного исследования CANTOS, длительное ингибирование выработки ИЛ-1 $\beta$ приводило к уменьшению как частоты приступов артрита у пациентов с подагрой, так и вероятности дебюта подагры в случае асимптоматической ГУ, причем вне зависимости от исходного уровня МК сыворотки [57]. Однако поскольку ГУ является основным фактором риска развития подагры [7], снижение МК, скорее всего, является главным фактором, объясняющим связь между бариатрической операцией и уменьшением риска развития подагры у лиц с ожирением.
Одним из наиболее крупных исследований по бариатрической хирургии является Шведское исследование ожирения - Swedish Obese Subjects study (SOS), где наблюдалось 4047 пациентов: 2010 человек, подвергнутых различным бариатрическим вмешательствам, и 2037 пациентов - контрольной группы (с 1987 по 2001 гг.). Продемонстрировано значимое снижение сопутствующих заболеваний у пациентов с ожирением, включая ГУ [47]. Авторы обнаружили, что проведение бариатрического вмешательства связано со снижением на 78\% и 51\% вероятности развития ГУ в сравнении с модификацией образа жизни через 2 и 10 лет после операции соответственно. После проведения бариатрического вмешательства уровень МК стал меньше на 14\% через 2 года $(n=1845)$ и на 8\% через 10 лет $(n=641)$ по сравнению с контрольными группами.-

C. Maglio и коллеги, основываясь на результатах шведского исследования, показали, что бариатрические операции предотвращают развитие ГУ у субъектов с исходно нормальным уровнем сывороточной МК до оперативного вмешательства. В исследование были включены 1982 пациентов, которым было проведено бариатрическое вмешательство, и контрольная группа из 1999 пациентов. Ни у одного из данной выборки пациентов не было подагры на момент начала SOS-исследования. Появление новых случаев подагры отмечалось согласно данным медицинской документации и национального регистра пациентов - National Patient Register, а также регистра назначаемых препаратов - Prescribed Drug Register (где учитывались установка диагноза подагры согласно международной классификации болезней, а также назначение аллопуринола и колхицина). Критерием ГУ был определен уровень МК более 6,8 мг/дл (>408 мкмоль/л). В хирургической группе 372 субъекта перенесли регулируемое бандажирование желудка, 1347 - продольную резекцию желудка и 263 - желудочное шунтирование. Всего было зафиксировано 339 случаев дебюта подагры (из которых 279 согласно регистру препаратов). Из них 128 пришлось на бариатрическую группу и 201 - на группу контроля. Все три вида бариатрических операций были ассоциированы с более низкой частотой развития подагры в сравнении с контрольной группой [58]. Используя кривую Kaplan-Meier, разница абсолютного риска развития подагры между бариатрической и контрольной группами составила 3 процентных пункта за 15 лет, а абсолютный риск развития Гу у лиц, ранее не имевших ее, составил $12 \%$ за 15 лет.

Тем не менее исследование имело некоторые ограничения, поскольку данные из центров первичной медицинской помощи, куда также могут обращаться пациенты с подагрой, ввиду отсутствия доступа к ним, не были включены. Кроме того, данные регистра лекарственных препаратов могли упускать иные причины назначения аллопуринола и колхицина (синдром распада опухоли, периодические лихорадки, болезнь депонирования кристаллов пирофосфата кальция, болезнь Бехчета), которые, однако, встречаются значительно реже, чем подагра. Кроме того, вероятно, в рамках данного регистра невозможно было учесть факт возможного назначения пациентам с острым подагрическим артритом глюкокортикоидов и НПВП. Другим существенным ограничением являлось то, что исследование кристаллов МУН редко 
использовалось в клинической практике, и в большинстве случаев диагноз устанавливался на основании клинико-лабораторных данных [59]. Следует также отметить, что около 70\% участников исследования SOS - женщины. Однако все ограничения данного исследования в равной степени распространялись как на бариатрическую, так и на контрольную группу.

N. Dalbeth и соавт. сравнивали влияние нехирургической потери веса и бариатрической хирургии на уровень МК у пациентов с морбидным ожирением и диабетом. В исследование вошли 60 человек с СД2 и индексом массы тела $\geq 35$ кг/м². Из всех пациентов 12 имели подагру. Средний вес участников до начала исследования составлял 135,3 кг. Примерно 40\% получали диуретики. После 6 месяцев лечения ожирения путем модификации образа жизни (гипокалорийная диета, расширение физических нагрузок) у всех участников была проведена лапароскопическая рукавная резекция желудка с последующим 1 годом наблюдения. Участники в среднем потеряли $5,5( \pm 4,1)$ кг веса за 6 месяцев модификации образа жизни до операции и $34,3( \pm 11,1)$ кг после операции.

Концентрация МК сыворотки к концу наблюдения не изменилась после нехирургической потери веса $(0,38 \pm 0,09$ ммоль/л в начале исследования и 0,38 0,10 ммоль/л в последующем), увеличилась до 0,44 $( \pm 0,15)$ ммоль/л в ближайшем послеоперационном периоде, но уменьшилась до 0,30 $( \pm 0,08)$ ммоль/л через 1 год после операции. Из 48 участников без подагры с МК сыворотки выше уровня насыщения ( $\geq 0,41$ ммоль/л) было у 19 (40\%) в начале исследования и у 1 (2\%) через 1 год после операции. У участников исследования с подагрой уровень МК выше терапевтических целевых уровней ( $\geq 0,36$ ммоль/л) отмечался в 10 из 12 (83\%) случаев в начале исследования до оперативного вмешательства и в 4 из 12 случаев (33\%) через 1 год после операции. Потребность в уратснижающей терапии снизилась при этом с 75\% (9 из 12 пациентов) до 33\% (4 из 12 пациентов). Несмотря на сравнительно небольшой размер выборки, данная работа имеет важное значение для поиска возможных путей достижения ремиссии подагры без использования уратснижающих препаратов [29].

Важно отметить, что в исследовании N. Dalbeth выборка пациентов была лимитирована наличием СД2. Это примечательно, потому что влияние потери веса на уровень МК сыворотки может варьироваться в зависимости от наличия диабета, так как гипергликемия связана с более низкими показателями МК сыворотки, чем у лиц без диабета, вероятно из-за урикозурического эффекта гипергликемии [60]. Большое проспективное когортное исследование $(\mathrm{n}=10$ 000) показало, что по сравнению с пациентами без СД2 распространенность ГУ была на 63\% ниже у мужчин с СД2 [61]. Эти данные также могут объяснить более высокий исходный уровень МК у пациентов без СД2 (0,57 ммоль/л) в исследовании P. Dessein [28] в сравнении со средним уровнем МК у пациентов, имеющих СД2 и подагру (0,41 ммоль/л) в исследовании N. Dalbeth [29], даже несмотря на более высокие исходные уровни ИМТ в последнем (30,5 и 48,5 кг/м² соответственно).

Кроме того, данный механизм может также объяснить разную выраженность эффекта модификации образа жизни в данных двух исследованиях. Из 12 больных по- дагрой и СД2 бариатрическая операция привела к снижению МК на 0,08 ммоль/л от исходного значения. Это может говорить о том, что большая потеря веса в 34 кг после бариатрической операции преодолела предполагаемый порог урикозурических эффектов гипергликемии. Примечательно, что количественное снижение уровня МК при потере 34 кг после бариатрической хирургии среди данных пациентов аналогично (а возможно, и меньше) снижению МК при потере 7,7 кг путем модификации образа жизни у пациентов без диабета. Однако исследование N. Dalbeth было ограничено высокой этнической принадлежностью к маори и тихоокеанцам, без значительной сопутствующей патологии [62].

P. Richette и коллеги исследовали влияние потери веса на уровень МК крови и активность ксантиноксидазы среди 154 пациентов со средним индексом массы тела 47,9 кг/м², 81\% из которых - женщины, большей части пациентов проведено гастрошунтирование. У всех пациентов с потерей веса отмечалось снижение активности ксантиноксидазы, но отсутствовала прямая корреляция со снижением МK [54].

E. Menenakos и коллеги исследовали взаимосвязь концентрации МК со снижением массы тела после желудочного шунтирования у 55 пациентов. Уровень МК сыворотки и ИМТ измерялись до операции и в первый послеоперационный месяц и через год. Концентрация МК в предоперационном периоде имела значимую положительную корреляцию с процентом общей потери веса в первый послеоперационный месяц и год. Авторы предположили, что уровень МК в сыворотке крови может быть предиктором успешно проведенного желудочного шунтирования и помочь в прогнозировании выраженности послеоперационной потери веса, в отличие от значения предоперационного ИМТ [63]. То есть наличие ГУ предполагает лучший результат желудочного шунтирования. Среди предполагаемых причин авторы статьи называют исходно различные уровни грелина, пептида, именуемого «гормоном голода» и синтезируемого клетками желудка, концентрация которого обратно коррелирует с уровнем МК сыворотки в популяции. При ожирении уровень данного гормона ниже, а пациенты с предоперационным повышенным уровнем МК и, следовательно, более низкими уровнями грелина имеют большую потерю веса. Среди других возможных причин упоминается предположение, что пациенты с ожирением, страдающие MC, в большей степени способны регулировать свой вес за счет более быстрых изменений скорости метаболизма. Наиболее простым объяснением считается ожидаемое изменение рациона питания после операции, которое исходно может быть более «нездоровым» у пациентов с ГУ. Это может быть важно и для понимания причин хороших результатов оперативного лечения ожирения в отношении динамики сывороточного уровня МК у пациентов с подагрой.

Влияние бариатрических операций на развитие новых приступов артрита

Любое оперативное вмешательство является фактором риска развития острого приступа подагры. Ранний послеоперационный период, оперативное лечение онкологического заболевания, дегидратация, катаболическое состояние с поражением мышц, 
высокие предоперационные уровни МК и прекращение уратснижающей терапии представляют собой наиболее важные факторы риска острых приступов [1, 2]. Клинические исследования показали, что бариатрическая хирургия не является исключением, но при этом риск развития приступов снижается с течением времени после операции [64].

P. Antozzi и коллеги ретроспективно оценили 1240 пациентов, которые перенесли лапароскопическое шунтирование желудка, показав, что у пяти пациентов $(0,4 \%)$ до операции имел место диагноз подагры и у двух из них развился острый приступ после операции в ближайшем послеоперационном периоде [65].

J. Friedman и колл. изучили 411 пациентов, перенесших лапароскопическое желудочное шунтирование. Предоперационная заболеваемость подагрой составила 5,1\%. Из этих пациентов у 33,3\% развился острый приступ после операции. Однако ни у кого из исследуемых без диагноза подагры до операции не развивался острый подагрический артрит после оперативного лечения [66].

H. Romero-Talamás и колл. сравнивали 99 больных ожирением и подагрой, которым была выполнена бариатрическая операция, с 56 пациентами, страдающими подагрой и ожирением, которым проводили небариатрические хирургические абдоминальные вмешательства. Авторы обнаружили, что частота приступов подагры в первый месяц после операции была статистически значимо выше в бариатрической группе (17,5\% против 1,8\%). При этом общая частота приступов в бариатрической группе снизилась с 23,8\% до 8\% через 1 год после операции. В контрольной группе разницы в количестве приступов подагры не наблюдалось [67].

У одного из пациентов ранее упомянутого исследования N. Dalbeth наблюдался приступ острого подагрического артрита в контексте тяжелой гиперурикемии [29].

G. Katsogridaki и колл. исследовали 85 пациентов, которым была выполнена продольная резекция желудка. Всем участникам проводилось определение уровня МК, а также биоимпедансометрия для исследования содержания уровня воды в организме. В группе исследуемых субъектов распространенность ГУ до операции составляла 30,6\% (26 человек). Общая распространенность послеоперационной ГУ - 18,8\% (16 человек), из которых 12 человек - с ГУ на дооперационном этапе и 4 человека без ранее зарегистрированной ГУ. Из указанных 12 человек приступ подагры в послеоперационном периоде развился у 3 пациентов (25\%); в то же время у 3 пациентов без ГУ в послеоперационном периоде впервые зарегистрирован острый подагрический артрит. Таким образом, общая распространенность послеоперационного приступа подагры составила 7,06\%. Кроме того, пациенты, у которых развился артрит, по данным биоимпедансометрии имели более низкое общее содержание воды по сравнению с остальными [68].

Развитие приступа артрита у пациентов с подагрой в раннем послеоперационном периоде может быть обусловлено длительным голоданием, ускоренным катаболизмом после оперативного вмешательства, дегидратацией. Данные состояния могут приводить к повышению уровня МК сыворотки [64]. Отчасти в случае бариатрических операций повышение МК возможно как следствие массивного разрушения ткани желудка. Первые недели после операции представляют период, в котором происходят наибольшая потеря веса и значительная вариабельность уровня МК, аналогично процессам, которые происходят при инициации уратснижающей терапии, также способствующей увеличению риска острого приступа артрита в первые месяцы от начала лечения [69]. Кроме того, бариатрическая хирургия может оказывать выраженное влияние на почечную (и, возможно, экстраренальную) экскрецию мочевой кислоты.

Диета, используемая в периоперационном периоде у пациентов с ожирением, которая обычно основана на малом количестве углеводов и высоком содержании белка (обычно 1,2-1,5 г на кг идеального веса), в течение по крайней мере 2 недель до и после хирургического вмешательства также, вероятно, может провоцировать острый приступ артрита [70]. Низкоуглеводная диета вызывает мобилизацию жирных кислот из печени, что связано с повышенным сопутствующим кетогенным состоянием с образованием $\beta$-гидроксибутирата и ацетоацетата, которые ингибируют почечную экскрецию MK [71]. Наконец, ошибочная отмена аллопуринола или препаратов для профилактики приступов подагры, одновременный прием диуретиков перед операцией также могут увеличить риск обострений подагры.

Недавно было опубликовано описание клинического случая развития подагры после бариатрического вмешательства [72]. Заболевание развилось через два года после перенесенного лапароскопического регулируемого бандажирования желудка пациенту в возрасте 35 лет с морбидным ожирением, неконтролируемой артериальной гипертензией и наличием ГУ. Послеоперационная потеря массы тела на 30 кг за 8 месяцев

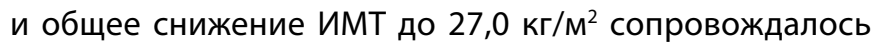
стойким повышением уровня МК, с максимальным значением за весь период наблюдения 825 мкмоль/л. Развитие подагры характеризовалась как стремительное, с рецидивирующими артритами и быстрым нарастанием тофусных масс. Функция почек при этом оставалась сохранной. В последующем, еще через 2 года, пациент был госпитализирован в связи с формированием бандаж-индуцированной перфорации желудка и перитонита, потребовавшего хирургического лечения, а также развитием острого почечного повреждения. После лечения, удаления бандажа отмечалась выраженная прибавка массы тела и дальнейшее прогрессирование подагры. Среди возможных факторов риска развития подагры - ускоренная потеря массы тела, высокобелковая диета, артериальная гипертензия и неалкогольная жировая болезнь печени. Учитывая результаты Шведского исследования [58], выбор данного вида операции, вероятно, не был определяющим фактором развития подагры, но его осложнения и необходимость оперативной коррекции привели к обострению. Можно предположить, что именно ускоренный распад белка, аденозинтрифосфата и нарастание ГУ при резкой потере массы тела привели к развитию подагры. Кроме того, любое оперативное вмешательство увеличивает вероятность развития подагрического приступа. При наличии противопоказаний к применению или неэффективности традиционных препаратов для купирования подагрического приступа возможно применение высокоэффективного ингибитора ИЛ-1 - канакинумаба [24]. 
В целом, вероятно, является целесообразным в рамках периоперационной подготовки бариатрических пациентов с подагрой производить достижение индивидуальных целевых показателей мочевой кислоты, при необходимости прием НПВП или колхицина для профилактики новых приступов артрита. Также желательно обеспечить адекватную гидратацию и отмену диуретиков (в отсутствие жизненных показаний). Важно отметить необходимость скорректированной диеты для бариатрических пациентов с учетом необходимости ограничения избыточного поступления пуринов, адекватного поступления углеводов и употребления продуктов, снижающих уровень МК. Ранняя реабилитация позволяет избежать длительной иммобилизации и связанных с ней последствий, таких как инфекции, респираторные осложнения, тромбоз глубоких вен и острые приступы подагры [55].

\section{ЗАКЛЮЧЕНИЕ}

Бариатрическая хирургия представляет собой эффективный метод лечения пациентов с морбидным ожирением, одним из важных отдаленных эффектов которой является уменьшение потребности или даже полная отмена уратснижающей терапии у пациентов с подагрой. Фактически на сегодняшний день - это наиболее эффективный немедикаментозный метод, при котором возможна ремиссия как СД2, так и подагры. Тем не менее особенности послеоперационного периода у этих больных, включающие высокий риск острых приступов артрита, предполагают необходимость назначения симптоматической противовоспалительной терапии, сохранения (возможно временного) приема уратснижающей терапии, а также адекватной гидратации. Учитывая актуальность проблемы, ограниченное количество исследований, имеется необходимость проведения дальнейших исследований по данному вопросу.

\section{ДОПОЛНИТЕЛЬНАЯ ИНФОРМАЦИЯ}

Источник финансирования. Подготовка и публикация рукописи проведены на личные средства авторского коллектива.

Конфликт интересов. Авторы декларируют отсутствие явных и потенциальных конфликтов интересов, связанных с публикацией настоящей статьи.

Участие авторов. Все авторы внесли значимый вклад в проведение исследования и подготовку статьи, прочли и одобрили финальную версию статьи перед публикацией.

\section{СПИСОК ЛИТЕРАТУРЫ | REFERENCES}

1. Насонова В.А., Барскова В.Г. Ранние диагностика и лечение подагры - научно обоснованное требование улучшения трудового и жизненного прогноза больных. // Научно-практическая ревматология. - 2004. - T. 42. - №1. - C. 5-7. [Nasonova VA, Barskova VG. Early diagnostic and treatment of gout - is scientifically based reguirements for improvement of labour and living prognosis of patients. Rheumatology Science and Practice. 2004;42(1):5-7. (In Russ.)] doi: https://doi.org/ 10.14412/1995-4484-2004-1374

2. Насонов Е.Л., Елисеев М.С. Роль интерлейкина 1 в развитии заболеваний человека. // Научно-практическая ревматология. 2016. — T. 54. — №1. — C. 60-77. [Nasonov EL, Eliseev MS. Role of interleukin 1 in the development of human diseases. Rheumatology Science and Practice. 2016;54(1):60-77. (In Russ.)] doi: https://doi.org/ 10.14412/1995-4484-2016-60-77

3. McQueen FM, Chhana A, Dalbeth N. Mechanisms of joint damage in gout: evidence from cellular and imaging studies. Nat Rev Rheumatol. 2012;8(3):173-181. doi: https://doi.org/ 10.1038/nrrheum.2011.207

4. Perez-Ruiz F, Dalbeth N, Bardin T. A review of uric acid, crystal deposition disease, and gout. Adv Ther. 2015;32(1):31-41. doi: https://doi.org/ 10.1007/s12325-014-0175-z

5. Loeb JN. The influence of temperature on the solubility of monosodium urate. Arthritis Rheum. 1972;15(2):189-192. doi: https://doi.org/ 10.1002/art.1780150209

6. Bardin T, Richette P. Definition of hyperuricemia and gouty conditions. Curr Opin Rheumatol. 2014;26(2):186-191. doi: https://doi.org/ 10.1097/BOR.0000000000000028

7. Campion EW, Glynn RJ, Delabry LO. Asymptomatic hyperuricemia. Risks and consequences in the normative aging study. Am J Med. 1987:82(3):421-426. doi: https://doi.org/ 10.1016/0002-9343(87)90441-4

8. Shiozawa A, Szabo SM, Bolzani A, et al. Serum Uric Acid and the Risk of Incident and Recurrent Gout: A Systematic Review. J Rheumatol. 2017:44(3):388-396. doi: https://doi.org/ 10.3899/jrheum.160452

9. Dalbeth N, Phipps-Green A, Frampton C, et al. Relationship between serum urate concentration and clinically evident incident gout: an individual participant data analysis. Ann Rheum Dis. 2018;77(7):1048-1052. doi: https://doi.org/ 10.1136/annrheumdis-2017-212288

10. Zuo T, Liu $X$, Jiang $L$, et al. Hyperuricemia and coronary heart disease mortality: a meta-analysis of prospective cohort studies. BMC Cardiovasc Dis. 2016;16(1). doi: https://doi.org/ 10.1186/s12872-016-0379-z
11. White J, Sofat R, Hemani G, et al. Plasma urate concentration and risk of coronary heart disease: a Mendelian randomisation analysis. Lancet Diabetes Endocrinol. 2016;4(4):327-336. doi: https://doi.org/ 10.1016/s2213-8587(15)00386-1

12. Елисеева М.Е., Елисеев М.С. Значение гиперурикемии в развитии заболеваний человека и методы ее коррекции. // Доктор.Ру. - 2019. — №2. — C. 47-54. [Eliseeva ME, Eliseev MS. The Role of Hyperuricemia in Human Morbidity, and Treatment Options. Doctor.Ru. 2019;(2):47-54. (In Russ.)] doi: https://doi.org/ 10.31550/1727-2378-2019-157-2-47-54

13. Bursill D, Taylor WJ, Terkeltaub R, et al. Gout, Hyperuricaemia and Crystal-Associated Disease Network (G-CAN) consensus statement regarding labels and definitions of disease states of gout. Ann Rheum Dis. 2019;78(11):1592-1600. doi: https://doi.org/ 10.1136/annrheumdis-2019-215933

14. Neogi T, Jansen TLTA, Dalbeth N, et al. 2015 Gout Classification Criteria: An American College of Rheumatology/European League Against Rheumatism Collaborative Initiative. Arthritis Rheumatol. 2015;67(10):2557-2568. doi: https://doi.org/ 10.1002/art.39254

15. Roddy E, Choi HK. Epidemiology of Gout. Rheum Dis Clin North Am. 2014;40(2):155-175. doi: https://doi.org/ 10.1016/j.rdc.2014.01.001

16. Zhu Y, Pandya BJ, Choi HK. Prevalence of gout and hyperuricemia in the US general population: The National Health and Nutrition Examination Survey 2007-2008. Arthritis Rheum. 2011;63(10):3136-3141. doi: https://doi.org/ 10.1002/art.30520

17. Kuo CF, Grainge MJ, Zhang W, Doherty M. Global epidemiology of gout: prevalence, incidence and risk factors. Nat Rev Rheumatol. 2015;11(11):649-662. doi: https://doi.org/ 10.1038/nrrheum.2015.91

18. Mikuls TR, Farrar JT, Bilker WB, et al. Gout epidemiology: results from the UK General Practice Research Database, 1990-1999. Ann Rheum Dis. 2005;64(2):267-272. doi: https://doi.org/ 10.1136/ard.2004.024091

19. Hyndman D, Liu S, Miner JN. Urate Handling in the Human Body. Curr Rheumatol Rep. 2016;18(6):34 doi: https://doi.org/ 10.1007/s1 1926-016-0587-7

20. Dalbeth N, House ME, Gamble GD, et al. Influence of the ABCG2 gout risk $141 \mathrm{~K}$ allele on urate metabolism during a fructose challenge. Arthritis Res Ther. 2014;16(1):R34. doi: https://doi.org/ 10.1186/ar4463

21. Chhana A, Lee G, Dalbeth N. Factors influencing the crystallization of monosodium urate: a systematic literature review. BMC Musculoskelet Disord. 2015;16:296. doi: https://doi.org/ 10.1186/s12891-015-0762-4 
22. Richette P, Doherty M, Pascual E, et al. 2018 updated European League Against Rheumatism evidence-based recommendations for the diagnosis of gout. Ann Rheum Dis. 2020;79(1):31-38. doi: https://doi.org/ 10.1136/annrheumdis-2019-215315

23. Khanna D, Fitzgerald JD, Khanna PP, et al. 2012 American College of Rheumatology guidelines for management of gout. Part 1: systematic nonpharmacologic and pharmacologic therapeutic approaches to hyperuricemia. Arthritis Care Res (Hoboken). 2012;64(10):1431-1446. doi: https://doi.org/ 10.1002/acr.21772

24. Елисеев МС. Подагра. В кн.: Ревматология. Российские клинические рекомендации. / Под ред. Насонова Е.Л. - М.: ГЭОТАР-Медиа; 2017. - С. 253-264. [Eliseev MS. Podagra. In: Nasovnov EL, editor. Revmatologiya. Russian clinical guidelines. Moscow: GEOTAR-Media; 2017. p. 253-264. (In Russ.)]

25. Choi HK, Curhan G. Soft drinks, fructose consumption, and the risk of gout in men: prospective cohort study. BMJ. 2008;336(7639):309-312. doi: https://doi.org/10.1136/bmj.39449.819271.BE

26. Барскова В.Г., Елисеев М.С., Насонова В.А., Насонов Е.Л. Алкоголь и клиническая картина у больных подагрой. // Современная ревматология. - 2007. - Т. 1. - №1. - С. 37-42. [Barskova VG, Yeliseyev MS, Nasonova VA, Nasonov YL. Alcohol and the clinical picture of gout. Sovremennaia revmatologiia. 2007;1 (1):37-42. (In Russ.)]

27. Li C, Hsieh MC, Chang SJ. Metabolic syndrome, diabetes, and hyperuricemia. Curr Opin Rheumatol. 2013;25(2):210-216. doi: https://doi.org/ 10.1097/BOR.0b013e32835d951e

28. Dessein PH, Shipton EA, Stanwix AE, et al. Beneficial effects of weight loss associated with moderate calorie/carbohydrate restriction, and increased proportional intake of protein and unsaturated fat on serum urate and lipoprotein levels in gout: a pilot study. Ann Rheum Dis. 2000;59(7):539-543. doi: https://doi.org/ 10.1136/ard.59.7.539

29. Dalbeth N, Chen P, White M, et al. Impact of bariatric surgery on serum urate targets in people with morbid obesity and diabetes: a prospective longitudinal study. Ann Rheum Dis. 2014;73(5):797-802. doi: https://doi.org/ 10.1136/annrheumdis-2013-203970

30. Yamashita S, Matsuzawa Y, Tokunaga K, et al. Studies on the impaired metabolism of uric acid in obese subjects: marked reduction of renal urate excretion and its improvement by a low-calorie diet. Int J Obes. 1986;10(4):255-264

31. Serpa Neto A, Rossi FM, Valle LG, et al. Relation of uric acid with components of metabolic syndrome before and after Roux-en-Y gastric bypass in morbidly obese subjects. Ara Bras Endocrinol Metabol. 2011;55(1):38-45. doi: https://doi.org/ 10.1590/s0004-27302011000100005

32. Choi HK, Ford ES, Li C, Curhan G. Prevalence of the metabolic syndrome in patients with gout: the Third National Health and Nutrition Examination Survey. Arthritis Rheum. 2007;57(1):109-115. doi: https://doi.org/ 10.1002/art.22466

33. Isomaa B, Almgren P, Tuomi T, et al. Cardiovascular morbidity and mortality associated with the metabolic syndrome. Diabetes Care. 2001;24(4):683-689. doi: https://doi.org/ 10.2337/diacare.24.4.683

34. Ротарь О.П., Либис Р.А., Исаева Е.Н., и др. Распространенность метаболического синдрома в разных городах РФ. // Российский кардиологический журнал. - 2012. - Т. 17. — №2. - С. 55-62. [Rotar OP, Libis RA, Isaeva EN, et al. Metabolic syndrome prevalence in Russian cities. Russian Journal of Cardiology. 2012;17(2):55-62. (In Russ.)] doi: https://doi.org/ 10.15829/1560-4071-2017-8-82-89

35. Kim TH, Lee SS, Yoo JH, et al. The relationship between the regional abdominal adipose tissue distribution and the serum uric acid levels in people with type 2 diabetes mellitus. Diabetol Metab Syndr. 2012:4(1):3. doi: https://doi.org/ 10.1186/1758-5996-4-3

36. Vuolteenaho K, Koskinen A, Moilanen E. Leptin - a link between obesity and osteoarthritis. applications for prevention and treatment. Basic Clin Pharmacol Toxicol. 2014;114(1):103-108. doi: https://doi.org/ 10.1111/bcpt.12160

37. Quinones Galvan A, Natali A, Baldi S, et al. Effect of insulin on uric acid excretion in humans. Am J Physiol. 1995;268(1 Pt 1):E1-5. doi: https://doi.org/ 10.1152/ajpendo.1995.268.1.E1

38. Huang J, Sun Y, Niu K, et al. Does elevated serum uric acid level predict the hypertension incidence? A Chinese prospective cohort study. Clin Exp Hypertens. 2015;37(6):498-504. doi: https://doi.org/ 10.3109/10641963.2015.1013121

39. Ma LD, Sun RX, Xin Y, et al. Clinical characteristics in gout patients with different body mass index. Zhonghua Nei Ke Za Zhi. 2017;56(5):353-357. doi: https://doi.org/ 10.3760/cma.j.issn.0578-1426.2017.05.010
40. Барскова ВГГ, Елисеев М.С., Зилов А.В., Насонов ЕЛ. Влияние гипергликемии и гиперинсулинемии на уровень мочевой кислоты и течение артрита у больных подагрой с сахарным диабетом 2 типа. // Ожирение и метаболизм. 2007. - T. 4. — №1. - C. 19-23. [Barskova VG, Eliseev MS, Zilov AV, Nasonov EL. Vliyanie giperglikemii i giperinsulinemii na uroven' mochevoy kisloty i techenie artrita u bol'nykh podagroy s sakharnym diabetom 2 tipa. Obesity and metabolism. 2007;4(1):19-23 (In Russ.)] doi: https://doi.org/ 10.14341/2071-8713-4908

41. Елисеев М.С., Барскова В.Г,, Ильиных Е.В., Насонова В.А. Синдром инсулинорезистентности и подагра: исторический аспект и современное состояние проблемы. // Клиническая геронтология. — 2005. - Т. 11. — №4. - C. 30-41. [Eliseev MS, Barskova VG, Il'inykh EV. Sindrom insulinorezistentnosti i podagra: istoricheskiy aspekt i sovremennoe sostoyanie problemy. Klinicheskaia gerontologiia. 2005;11(4):30-41. (In Russ.)]

42. Елисеев М.С., Барскова В.Г., Денисов И.С. Динамика клинических проявлений подагры у мужчин (данные 7-летнего ретроспективного наблюдения). // Терапевтический архив. 2015. - T. 87. — №5. - C. 10-15. [Eliseev MS, Barskova VG, Denisov IS. Time course of changes in the clinical manifestations of gout in men: Data of a 7-year retrospective follow-up. Ter Arkh.2015;87(5):10-15. (In Russ.)] doi: https://doi.org/ 10.17116/terarkh201587510-15

43. Ellmann H, Bayat S, Araujo E, et al. Effects of Conventional Uric AcidLowering Therapy on Monosodium Urate Crystal Deposits. Arthritis Rheumatol. 2020;72(1):150-156. doi: https://doi.org/ 10.1002/art.41063

44. Nielsen SM, Bartels EM, Henriksen M, et al. OP0340 Weight loss for overweight and obese individuals with gout: a systematic review of longitudinal observational studies. Ann Rheum Dis. 2017;76(Suppl 2):191-196. doi: https://doi.org/ 10.1136/annrheumdis-2017-eular.2651

45. Singh M. Mood, food, and obesity. Front Psychol. 2014;5. doi: https://doi.org/ 10.3389/fpsyg.2014.00925

46. Treatment of obesity: drugs and surgery. In: Powers PS. Obesity: the regulation of weight. Baltimore: Williams \& Wilkins; 1980. p. 325-389.

47. Sjöström L, Lindroos AK, Peltonen M. Lifestyle, diabetes, and cardiovascular risk factors 10 years after bariatric surgery. J Vasc Surg. 2005;41(5):911. doi: https://doi.org/ 10.1016/j.jvs.2005.02.005

48. Lenz M, Richter T, Mühlhauser I. The Morbidity and Mortality Associated With Overweight and Obesity in Adulthood. Dtsch Arztebl Int. 2009. doi: https://doi.org/ 10.3238/arztebl.2009.0641

49. Buchwald H, Avidor Y, Braunwald E, et al. Bariatric Surgery. Jama. 2004;292(14):1724. doi: https://doi.org/ 10.1001/jama.292.14.1724

50. Дедов И.И., Мельниченко Г.А., Шестакова М.В., и др. Национальные клинические рекомендации по лечению морбидного ожирения у взрослых. 3-ий пересмотр (лечение морбидного ожирения у взрослых). // Ожирение и метаболизм. - 2018. - Т. 15. №1. - C. 53-70. [Dedov II, Melnichenko GA, Shestakova MV, et al. Russian national clinical recommendations for morbid obesity treatment in adults. 3rd revision (Morbid obesity treatment in adults). Obesity and metabolism. 2018;15(1):53-70. (In Russ.)] doi: https://doi.org/ 10.14341/OMET2018153-70

51. Fried M, Yumuk V, Oppert JM, et al. Interdisciplinary European Guidelines on metabolic and bariatric surgery. Obes Facts. 2013;6(5):449-468. doi: https://doi.org/ 10.1159/000355480

52. O'Brien PE, Dixon JB, Laurie C, et al. Treatment of Mild to Moderate Obesity with Laparoscopic Adjustable Gastric Banding or an Intensive Medical Program. Ann Intern Med. 2006;144(9):625. doi: https://doi.org/ 10.7326/0003-4819-144-9-200605020-00005

53. Facchini F. Relationship Between Resistance to InsulinMediated Glucose Uptake, Urinary Uric Acid Clearance, and Plasma Uric Acid Concentration. Jama. 1991;266(21):3008. doi: https://doi.org/ 10.1001/jama.1991.03470210076036

54. Richette P, Poitou C, Manivet P, et al. Weight Loss, Xanthine Oxidase, and Serum Urate Levels: A Prospective Longitudinal Study of Obese Patients. Arthritis Care Res (Hoboken). 2016;68(7):1036-1042. doi: https://doi.org/ 10.1002/acr.22798

55. Tana C, Busetto L, Di Vincenzo A, et al. Management of hyperuricemia and gout in obese patients undergoing bariatric surgery. Postgrad Med. 2018;130(6):523-535. doi: https://doi.org/ 10.1080/00325481.2018.1485444

56. Dalbeth N, Pool B, Yip S, et al. Effect of bariatric surgery on the inflammatory response to monosodium urate crystals: a prospective study. Ann Rheum Dis. 2013;72(9):1583-1584. doi: https://doi.org/ 10.1136/annrheumdis-2013-203545 
57. Ridker PM, Howard CP, Walter $\mathrm{V}$, et al. Effects of interleukin-1 beta inhibition with canakinumab on hemoglobin A1c, lipids, C-reactive protein, interleukin-6, and fibrinogen: a phase $\mathrm{ll} b$ randomized, placebo-controlled trial. Circulation. 2012;126(23):2739-2748. doi: https://doi.org/ 10.1161/CIRCULATIONAHA.112.122556

58. Maglio C, Peltonen M, Neovius M, et al. Effects of bariatric surgery on gout incidence in the Swedish Obese Subjects study: a non-randomised, prospective, controlled intervention trial. Ann Rheum Dis. 2017;76(4):688-693. doi: https://doi.org/ 10.1136/annrheumdis-2016-209958

59. Zhang W. EULAR evidence based recommendations for gout. Part I: Diagnosis. Report of a task force of the standing committee for international clinical studies including therapeutics (ESCISIT). Ann Rheum Dis. 2006;65(10):1301-1311. doi: https://doi.org/ 10.1136/ard.2006.055251

60. Паневин Т.С., Елисеев М.С., Шестакова М.В., Насонов Е.Л. Преимущества терапии ингибиторами натрий-глюкозного котранспортера 2 типа у пациентов с сахарным диабетом 2 типа в сочетании с гиперурикемией и подагрой. // Терапевтический архив. - 2020. - Т. 92. — №5. - C. 110-118. [Panevin TS, Eliseev MS, Shestakova MV, Nasonov EL. Advantages of therapy with sodium glucose cotransporter type 2 inhibitors in patients with type 2 diabetes mellitus in combination with hyperuricemia and gout. Ter Arkh. 2020;92(5):110-118. (In Russ.)] doi: https://doi.org/ 10.26442/00403660.2020.05.000633

61. Herman J, Goldbourt U. Uric Acid and Diabetes: Observations in a Population Study. Lancet. 1982;320(8292):240-243. doi: https://doi.org/ 10.1016/s0140-6736(82)90324-5

62. Richette P, Poitou C, Garnero P, et al. 005 Benefits of Massive Weight Loss on Symptoms, Systemic Inflammation and Cartilage Turnover in Obese Patientswith Knee Osteoarthritis. Osteoarthritis Cartilage. 2010;18:S11. doi: https://doi.org/ 10.1016/s1063-4584(10)60032-5

63. Menenakos E, Doulami G, Tzanetakou IP, et al. The Use of Serum Uric Acid Concentration as an Indicator of Laparoscopic Sleeve Gastrectomy Success. Int Surg. 2015;100(1):173-179. doi: https://doi.org/ 10.9738/intsurg-d-13-00186.1

64. Craig MH, Poole GV, Hauser CJ. Postsurgical gout. Am Surg. 1995;61(1):56-59.
65. Antozzi P, Soto F, Arias F, et al. Development of Acute Gouty Attack in the Morbidly Obese Population after Bariatric Surgery. Obes Surg. 2005;15(3):405-407. doi: https://doi.org/ 10.1381/0960892053576802

66. Friedman JE, Dallal RM, Lord JL. Gouty attacks occur frequently in postoperative gastric bypass patients. Surg Obes Relat Dis. 2008;4(1):11-13. doi: https://doi.org/ 10.1016/j.soard.2007.09.012

67. Romero-Talamas $\mathrm{H}$, Daigle $\mathrm{CR}$, Aminian A et al. The effect of bariatric surgery on gout: a comparative study. Surg Obes Relat Dis. 2014;10(6):1161-1165. doi: https://doi.org/ 10.1016/j.soard.2014.02.025

68. Katsogridaki G, Tzovaras G, Sioka E, et al. Hyperuricemia and acute gout after laparoscopic sleeve gastrectomy. Clin Obes. 2019;9(2):e12296. doi: https://doi.org/ 10.1111/cob.12296

69. Чикина М.Н. Профилактика приступов артрита при назначении уратснижающей терапии у больных подагрой. // Научно-практическая ревматология. - 2018. - Т. 56. №6. - C. 760-766. [Chikina MN. Prevention of arthritis attacks in the use of urate-lowering therapy in patients with gout. Rheumatology Science and Practice. 2018;56(6):760-766. (In Russ.)] doi: https://doi.org/ 10.14412/1995-4484-2018-760-766

70. Busetto L, Marangon M, De Stefano F. High-protein lowcarbohydrate diets: what is the rationale? Diabetes Metab Res Rev. 2011;27(3):230-232. doi: https://doi.org/ 10.1002/dmrr.1171

71. Busetto L, Dicker D, Azran C, et al. Practical Recommendations of the Obesity Management Task Force of the European Association for the Study of Obesity for the Post-Bariatric Surgery Medical Management. Obes Facts. 2017;10(6):597-632. doi: https://doi.org/ 10.1159/000481825

72. Кушнаренко Н.Н., Мишко М.Ю., Медведева Т.А., Кушнаренко К.Е. Случай развития хронической тофусной подагры у пациента с морбидным ожирением после лапароскопического регулируемого бандажирования желудка. // Ожирение и метаболизм. - 2020. - Т. 17. — №1. - C. 93-99. [Kushnarenko NN, Mishko MY, Medvedeva TA, Kushnarenko KE. A case of chronic tophi gout in morbidly obese patient after laparoscopic adjustable gastric banding. Obesity and metabolism. 2020;17(1):93-99. (In Russ.)] doi: https://doi.org/ 10.14341/omet9736

\section{ИНФОРМАЦИЯ ОБ АВТОРАХ [AUTHORS INFO]:}

*Паневин Тарас Сергеевич [Taras S. Panevin, MD]; адрес: Россия, 115522, Москва, Каширское шоссе, д. 34A [address: 34A Kashirskoe Shosse, 115522 Moscow, Russia]; ORCID: https://orcid.org/0000-0002-5290-156X; eLibrary SPIN-код: 7839-3145; e-mail: tarasel@list.ru

Елисеев Максим Сергеевич, к.M.H. [Maxim S. Eliseev, MD, PhD]; ORCID: https://orcid.org/0000-0003-1191-5831; eLibrary SPIN: 2524-7320; e-mail: elicmax@rambler.ru

Шестакова Марина Владимировна, д.М.н., професcop [Marina V. Shestakova, MD, PhD. Professor]; ORCID: https://orcid.org/0000-0002-5057-127X; eLibrary SPIN: 3482-7651; e-mail: nephro@endocrincentr.ru

*Автор, ответственный за переписку / Corresponding author.

\section{ЦИТИРОВАТЬ:}

Паневин Т.С., Елисеев М.С., Шестакова М.В. Влияние хирургического лечения ожирения на пуриновый обмен и подагру // Ожирение и метаболизм. - 2020. - Т. 17. - №2. - С. 138-146. doi: https://doi.org/10.14341/omet12109

\section{TO CITE THIS ARTICLE:}

Panevin TS, Eliseev MS, Shestakova MV.The effect of bariatric surgery on purine metabolism and gout. Obesity and metabolism. 2020;17(2):138-146. doi: https://doi.org/10.14341/omet12109 